\title{
Estudio comparativo del daño renal producido tras la litotricia según la localización litiásica
}

\author{
Ma $^{\mathrm{a}}$ J. Cancho Gil, R. Díz Rodríguez, M. Vírseda Chamorro, C. Alpuente Román, \\ J.A. Cabrera Cabrera, P. Paños Lozano
}

Servicio de Urología. Hospital Central de la Defensa. Madrid.

Actas Urol Esp 2005; 29 (4): 373-377

\section{RESUMEN}

ESTUDIO COMPARATIVO DEL DAÑO RENAL PRODUCIDO TRAS LA LITOTRICIA SEGÚN LA LOCALIZACIÓN LITIÁSICA

Introducción: La litroticia por ondas de choque (LEOC) es fundamental en el tratamiento de las litiasis. Sin embargo, existen evidencias de que puede producir daño renal. El objetivo de nuestro estudio es determinar el grado de afectación de la función glomerular y tubular tras LEOC, y si influye la localización litiásica en el tipo de daño renal.

Material y métodos. Se realizó un estudio longitudinal prospectivo en 14 pacientes con función renal normal sometidos a LEOC. Se determinó el nivel basal, y a las 24 horas, al $4^{\circ}$ y al $10^{\circ}$ día post LEOC, de microalbuminuria (MA) (que valora la función glomerular), y de N-acetil glucosamida (NAG) y la alanina aminopeptidasa (AAP) (que valoran la función tubular).

Resultados: Los niveles de basales de MA, NAG y AAP no mostraron diferencias significativas en relación con la localización de los cálculos. Se observó un aumento significativo de los tres parámetros sólo a las 24 horas post LEOC. No se observaron diferencias significativas en la variación de los niveles de microalbuminuria, AAP y NAG con el tratamiento en relación a la localización de los cálculos.

Conclusiones: Existe un daño glomerular y tubular tras LEO. Este daño es independiente de la localización pélvica o calicial del cálculo. En pacientes con función renal normal previa, el daño renal se recupera al $4^{\circ}$ día post LEOC.

Palabras clave: Litotricia extracorpórea por ondas de choque. Litiasis renal. Lesión renal. Función glomerular. Función tubular.

\section{ABSTRACT}

A COMPARATIVE STUDY OF THE RENAL DAMAGE PRODUCED AFTER THE EXTRACORPOREAL SHOCK WAVE LITHOTRIPSY ACCORDING TO THE LITHIASIS LOCATION

Introduction: The Extracorporeal shock waves lithotripsy (ESWL) is fundamental in the treatment of lithiasis. However, there are evidences that it can produce renal damage. The objective of our study is to determine the degree of affectation of the glomerular and tubular function after LEOC, and the influence of the lithiasis location on the type of renal damage.

Material and methods: A prospective longitudinal study was carried out in 14 patients with normal renal function subjected to ESWL. We determined the basal level, and the levels at the 24 hours, at the 4 th and the 10th day post ESWL of: microalbuminuria (MA) (that values the glomerular function), and N-acetil glucosamide (NAG) and alanine aminopeptidase (AAP), (that value the tubular function).

Results: The basal levels of of MA, NAG and AAP didn't show significant differences in connection with the localization of the stones. A significant increase was observed of the three parameters only 24 hours post ESWL No significant differences were observed between the variation of the microalbuminuria levels, AAP and NAG and the treatment in relation to the localization of the stones.

Conclusions: It exists a glomerular and tubular damage after ESWL. This damage is not related with the pelvic or calicial location of the stones. In patient with previous normal renal function, the renal damage recovers at the $4^{\circ}$ day post ESWL.

Keywords: Extracorporeal Shock Waves Lithotripsy. Renal lithiasis. Renal damage. Glomerular function. Tubular function.

$\mathrm{U}$ na de las patologias más antiguas sobre la que poseemos documentación es la calculosis de las vías urinarias. Esta patología está documentada en una momia hallada en una tumba prehistórica y predinástica, descubierta en Egipto, que perteneció a un joven de unos 15 años que vivió unos 5.000 años antes de Cristo ${ }^{1}$.
Desde entonces y hasta nuestros días han sido innumerables las técnicas terapéuticas propuestas para el tratamiento más eficaz de estas litiasis. La introducción en 1982 por Chaussy de la litotricia extracorpórea por ondas de choque en la clínica diaria, supuso un cambio radical en el enfoque terapéutico de las litiasis del aparato uri- 
nario ${ }^{2}$. En actualidad la litotricia extracorpórea por ondas de choque (LEOC) constituye el tratamiento de primera elección para la gran mayoría de los casos de litiasis renales menores de 2 centímetros con independencia de su localización o composición ${ }^{3}$. Sin embargo a pesar de considerarse una terapia segura, existen evidencias de que puede producir diversas lesiones en el parénquima renal, cuya repercusión clínica no ha sido hasta el momento completamente esclarecida. La creciente tendencia a repetir las sesiones de litotricia en los casos en los que el primer tratamiento no fue totalmente eficaz, aumenta la importancia del conocimiento de estas lesiones, asî como del tiempo que tardan las células renales en recuperarse. Algunas lesiones macroscópicas se han relacionado con el empleo de la litotricia como las macro y microhematuria, los hematomas renales subcapsulares, las hemorragias lineales intratubulares, las áreas de necrosis focales, las obstrucciones de las vías urinarias, las hipertensiones o las sepsis urinarias ${ }^{4}$. Pero existe todavía un considerable desconocimiento sobre las lesiones ultramicroscópicas a nivel celular y sobre las alteraciones renales funcionales que pueden suceder tras el empleo de esta técnica.

La medición en la orina de ciertos marcadores bioquímicos constituye un método muy sensible para evaluar las lesiones del parénquima renal. Puesto que las distintas enzimas se correlacionan de forma específica con distintos tipos celulares del tejido renal, los cambios en su concentración urinaria pueden ser orientativos hacía la localización de las lesiones. Algunos de estos marcadores han sido aplicados hasta el momento para evaluar la incidencia de lesión renal post litotricia: alanina aminotransferasa (ALT), aspartato ami- notransferasa (AST), $\beta_{2}$ microglobulina, Gamma glutamil transpepsidasa (GGT), lactato deshidrogenasa (LDH), N-acetil glucosamina, alanino aminopeptidasa (AAP), acetil colinesterasa, leucina aminopeptidasa (LAP), kalicreina y $\beta$ galactosidasa, son algunos ejemplos ${ }^{5}$.

En el presente trabajo, hemos pretendido determinar la localización del daño renal tras una sesión de litotricia (glomerular o tubular), valorar si el daño renal varia según la localización litiásica (pélvica o calicial) y definir el tiempo que tarda el riñón en recuperarse por completo de dicho daño.

Para nuestro estudio nos basamos en tres parámetros: la microalbuminuria cuya detección en orina a dosis mas elevadas es un método bioquímico muy sensible para valorar la integridad de la membrana glomerular y en la N-acetil glucosamida (NAG) y la alanina aminopeptidasa (AAP) que reflejan con su elevación de forma muy específica el daño de las células del túbulo renal.

\section{MATERIAL Y MÉTODOS}

Se realizó un estudio longitudinal prospectivo en una cohorte compuesta por catorce pacientes, ocho varones y seis mujeres afectos de litiasis renal y que iban a ser tratados con litotricia extracorpórea por ondas de choque (LEOC). La edad media de la cohorte fue de 48,9 años, con intervalos de edad entre los 32 y los 70 años. Los criterios de inclusión y exclusión en el estudio se muestran en la Tabla 1.

Las variables a estudio fueron la microalbuminuria, y las concentraciones urinarias de $\mathrm{N}$-acetil glucosamida (NAG) y de alanino-amino-peptidasa (AAP). La primera determinación se realizó previamente al tratamiento. La segunda determinación fue realizada 24 horas después del trata-

Tabla 1

Criterios de inclusión y exclusión en el estudio

Criterios de inclusión

Edad del paciente entre 20 y 70 años.

Litiasis renal única no superior a 2 centímetros.

Litiasis oxálicas.

Función renal del paciente normal previa al tratamiento.

Litiasis que se fragmento en una sola sesión.

\section{Criterios de exclusión}

Infecciones urinarias de repetición.

Enfermedades sistémicas (diabetes, lupus, etc.).

Pacientes sometidos con anterioridad a cirugías del aparato urinario

Pacientes portadores de otras litiasis urinarias

Pacientes que requirieron algún tipo de maniobra endourológica (catéteres doble J, ureteroscopias, etc.).

Los pacientes cuyo cálculo producía hidronefrosis renal 
miento con litotricia. La tercera y la cuarta determinaciones se realizaron respectivamente el cuarto y el décimo día.

La orina de cada muestra se centrifugó a 2.000 r.p.m. durante cinco minutos y se almacenó a $-20^{\circ} \mathrm{C}$, hasta la realización de las técnicas analíticas correspondientes. Cada muestra de orina correspondía a la totalidad de la orina de la primera micción de la mañana, medida en mililitros, lo que se corresponde a un periodo de excreción urinaria de 6-8 horas.

Las técnicas de laboratorio empleadas para la cuantificación de las determinaciones fueron las siguientes: La microalbuminuria (MA) se cuantificó mediante el autoanalizador Beckman CX7 siguiendo los métodos de turbimetría. La alanino-amino-peptidasa (AAP) y la N-acetil-glucosamina (NAG) se midió con el espectrofotometro de Kontron "Uvicon 922", siendo la técnica de medición la colorimetría. El nivel de microalbumminuria se expresó en microgramos /minuto, y el de AAP y NAG en UI/litro.

El tratamiento (LEOC) se efectuó con un litotritor Compact Delta Dornier, con generador electromagnético y control radiológico. Los parámetros fijos para el tratamiento se muestran en la Tabla 2. Los pacientes permanecieron en ayunas las 6 horas previas al tratamiento. No se utilizó analgesia ni fluidoterapia durante la sesión de LEOC, aunque en algunos casos se realizó la sedación del paciente.

Para el análisis estadístico de los datos obtenidos se utilizó el programa SPSS-PC de la Universidad de Washington. El ajuste de la muestra a una distribución normal, según el test de Kolmogorov-Smirnov. Una vez confirmado el ajuste de la muestra a una distribución normal, se efectuaron los análisis de las muestras según el test

Tabla 2

Parámetros del tratamiento de la litiasis por LEOC

\begin{tabular}{ll}
\hline Parámetros de la litotricia & \\
\hline $\begin{array}{l}\text { Número de impactos } \\
\text { por sesión de tratamiento }\end{array}$ & 4.000 impactos \\
$\begin{array}{l}\text { Frecuencia de impactos. } \\
\begin{array}{l}\text { Potencia de la onda } \\
\text { de choque. }\end{array}\end{array}$ & No-100 impactos/minuto. \\
$\begin{array}{l}\text { Tiempo aproximado } \\
\text { por tratamiento. }\end{array}$ & 45 minutos.
\end{tabular}

paramétrico de comparación de las medias de la t de Student para datos pareados. El nivel de significación alfa se fijó en el 0,05 bilateral.

\section{RESULTADOS}

\section{Estadistica descriptiva}

La distribución de la muestra atendiendo a la localización de los cálculos fue de ocho pacientes con litiasis renal derecha y seis restantes pacientes con litiasis renal izquierda. Respecto a su posición dentro del riñón, siete pacientes portaban su litiasis dentro de un cáliz, y otros siete pacientes tenían el cálculo en la pelvis renal.

La aplicación a la muestra del test de Kolmogorov-Smirnov, nos indicó que todos los datos obtenidos de las variables MA, NAG y AAP en sus diferentes muestras, se ajustaron a una distribución normal, por lo que pudieron ser tratados mediante test paramétricos.

Análisis comparativo de los resultados

Los niveles basales de las variables MA, NAG y AAP no mostraron diferencias significativas en relación con la localización de los cálculos.

La variación de los niveles de microalbuminuria con el tratamiento se muestran en la Figura 1. Únicamente se observaron diferencias significativas respecto a su valor basal en la determinación inmediatamente posterior a la litotricia.

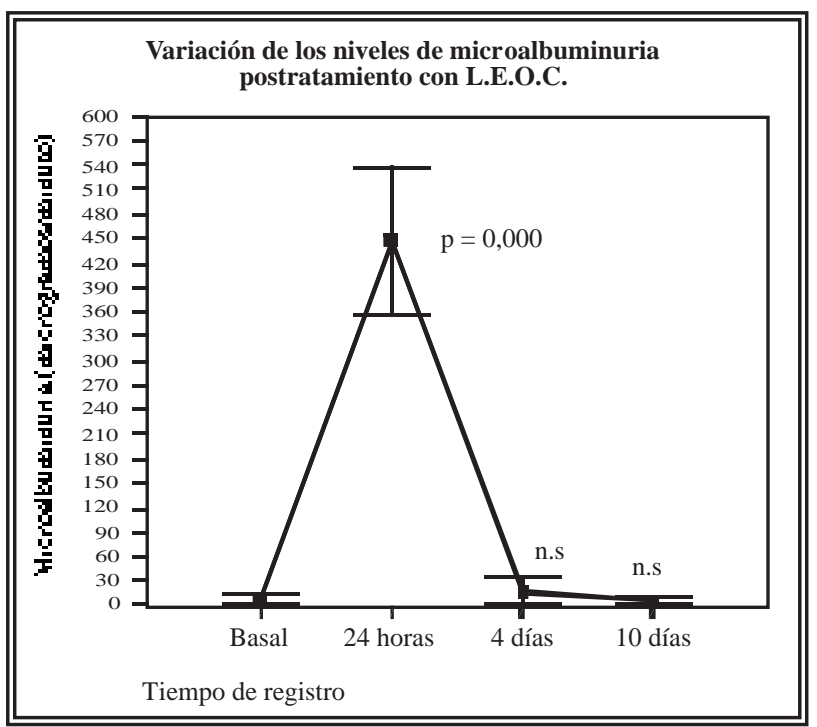

FIGURA 1. Diagrama de barras de error. El cuadrado central representa el valor de la media, y la longitud de los segmentos es proporcional a la desviación tipica. Se observa una elevación significativa de la microalbuminuria únicamente en las $\mathbf{2 4}$ horas posteriores al tratamiento. 
La variación de los niveles de NAG con el tratamiento se muestran en la Figura 2. Únicamente se observaron diferencias significativas respecto a su valor basal en la determinación inmediatamente posterior a la litotricia.

La variación de los niveles de AAP con el tratamiento se muestran en la Figura 3. Únicamente se observaron diferencias significativas respecto a su valor basal en la determinación inmediatamente posterior a la litotricia.

No se observaron diferencias significativas en la variación de los niveles de microalbuminuria, AAP y NAG con el tratamiento en relación a la localización de los cálculos.

\section{DISCUSIÓN}

Según los resultados obtenidos en nuestra experiencia, tras el estudio comparativo de los valores medios iniciales de los marcadores estudiados tanto en la pelvis como en los cálices, se aprecia una modificación estadísticamente significativa entre los parámetros obtenidos en la primera muestra post LEOC, que nos permite afirmar que la litotricia produce un daño renal a nivel glomerular y tubular tanto la litiasis se sitúe en la pelvis renal o en alguno de sus cálices.

El marcador utilizado para la detección de

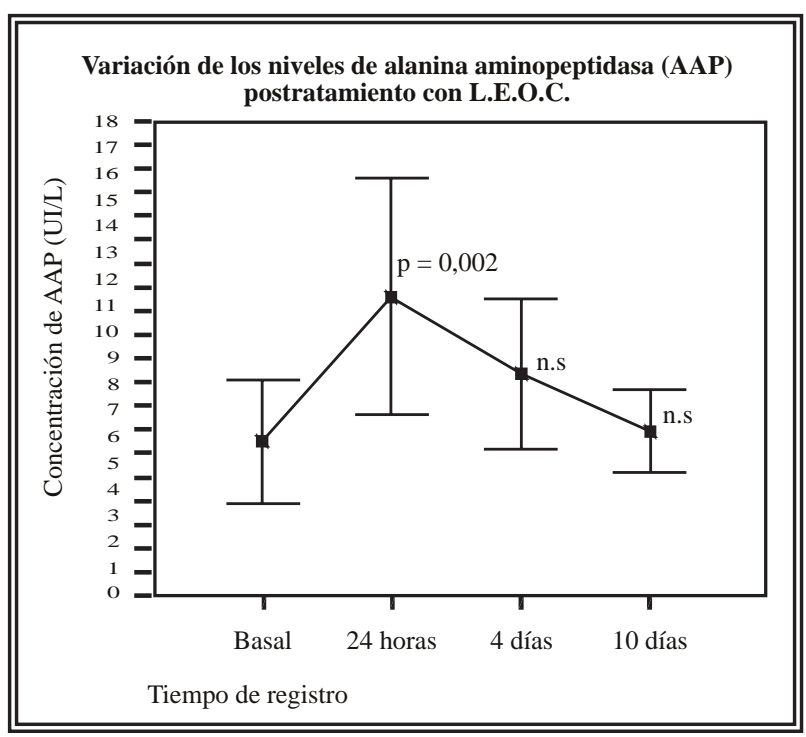

FIGURA 2. Diagrama de barras de error. El cuadrado central representa el valor de la media, y la longitud de los segmentos es proporcional a la desviación típica. Se observa una elevación significativa de la concentración de alanina aminopeptidasa (AAP) únicamente en las 24 horas posteriores al tratamiento.

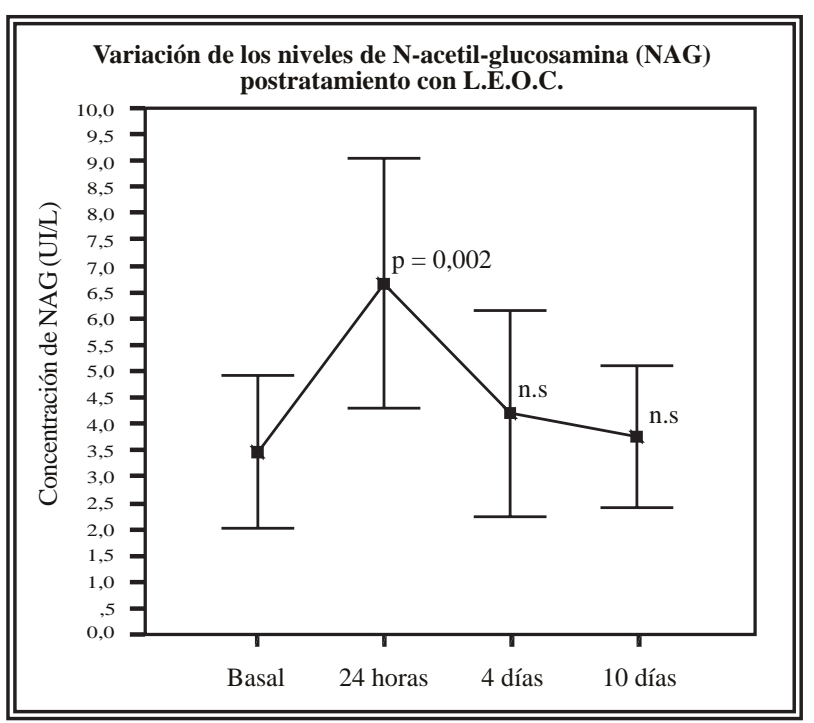

FIGURA 3. Diagrama de barras de error. El cuadrado central representa el valor de la media, y la longitud de los segmentos es proporcional a la desviación típica. Se observa una elevación significativa de la concentración de N-acetil-glucosamina (NAG) únicamente en las 24 horas posteriores al tratamiento.

lesión en el glomérulo, la microalbuminuria, se eleva de forma muy marcada a las 24 del tratamiento para normalizarse en torno al cuarto día. Las enzimas utilizadas para la detección del daño tubular, la AAP y la NAG, por el contrario se elevan de una manera más discreta al primer día de tratamiento pero permanecen elevadas aún al cuarto día y comienzan su normalización al décimo día. Aunque, de manera semejante a la microalbuminuria, sólo en el primer día del tratamiento existen diferencias estadísticamente significativas respecto a su valor basal.

La TEA (tasa de excreción de albúmina en orina) se sitúa entre 20 y 2000 microgramos/minuto. Diversos factores pueden influir en la excreción urinaria de albúmina: aumenta con los traumatismos, el ejercicio intenso, la sobrecarga de líquidos, el tratamiento oral con proteínas, las infecciones urinarias de vía alta, la HTA y el embarazo. Un aumento post-LEOC de albuminuria nos orientara sobre una alteración en la permeabilidad de la membrana glomerular así como del tiempo que permanece alterada dicha membrana tras el tratamiento. Sen et al, también encuentran un aumento transitorio de la albuminuria inmediatamente después de la litotricia que se normaliza rápidamente sin dejar secuelas ${ }^{6}$. 
Estudios experimentales en animales de laboratorio indican que el aclaramiento de inulina (parámetro que mide la filtración glomerular), se reduce transitoriamente en el periodo comprendido entre 1 y 4 horas post-litotricia para recuperarse posteriormente ${ }^{7}$. Sin embargo, esta alteración glomerular no suele detectarse si se utilizan pruebas menos sensibles como el aclaramiento de creatinina ${ }^{8,9}$.

$\mathrm{El}$ aumento de la excreción urinaria de las enzimas N-acetil-glucosamina (NAG) y de alanino-amino-peptidasa (AAP), nos mostrará el grado de daño que sufre la función tubular renal tras una sesión de litotricia, así como el tiempo de recuperación de esta alteración en la excreción tubular. Algunos autores también registran un incremento transitorio de $\mathrm{N}$-acetil glucosamida y otros marcadores asociados a lesión tubular, como la gamma-glutamiltransferrasa y la beta-2-microglobulina inmediatamente después de la litotricia ${ }^{6,8}$. Sin embargo, para otros autores no existe evidencia de elevación de la enzima NAG después del tratamiento con litotricia e incluso tras nefrostomía percutánea ${ }^{10}$.

No hay un criterio uniforme respecto al tiempo necesario para la normalización de estos parámetros. Algunos autores, tras analizar la concentración urinaria post LEOC de LAP y GGT reducen el tiempo de recuperación del daño tubular a 7 días $^{6}$, o bien encuentran una eliminación aumentada de albúmina en orina durante 7 dias, tiempo que fijan como necesario para la recuperación del daño glomerular tras la litotricia ${ }^{11}$. Para otros, habría una elevación de las enzimas tubulares hasta 30 días después de la litotricia ${ }^{12}$. Es posible que el tiempo necesario para la normalización de la función renal dependa de varios factores como: la existencia de infección urinaria, el número e intensidad de las ondas de choque, la presencia de anomalías renales preexistentes y la localización de los cálculos ${ }^{13}$. En nuestro estudio, la localización de los cálculos no mostró una relación significativa con el daño renal. Si bien es cierto, que aunque no alcanzó una significación estadística, si se observó una tendencia respecto a una mayor microalbuminuria post-litotricia en los pacientes con litiasis calicial.

En conclusión, podemos decir que:

1. Se produce un daño renal tanto a nivel glomerular como tubular tras el tratamiento de una litiasis con litotricia extracorpórea por ondas de choque.
2. Este daño es independiente de que el cálculo se sitúe en la pelvis renal o en uno de los cálices renales,

3. En nuestro grupo de pacientes seleccionados sin otra alteración renal que la litiasis, la recuperación tanto del filtrado glomerular, como de la excreción tubular se produce a partir de las 24 horas del tratamiento. Sin embargo, no se puede descartar que en pacientes con función renal deteriorada, este tratamiento origine una lesión renal más prolongada.

\section{REFERENCIAS}

1. Ayuso PP. Una historia de la Urología. Madrid. IM\&C, 1992

2. Martín I, Resnick MD. Litotripsia extracorpórea por ondas de choque; Desarrollo, instrumentos y situación actual. En: Clínicas de urología de norteamÉrica. Vol 1. Mexico: Interamericana, 1997.p. 205-229.

3. Ruiz Marcellán F.J. Litotricia extracorpórea por ondas de choque. En: Jiménez Cruz y Rioja Sanz. Tratado de urologia. Tomo I. Barcelona. Prous ediciones, 1993.p. 794-799.

4. Dominguez Molinero JF, Arrabal Martín M, Miján Ortiz JL, López Carmona F, de la Fuente Serrano A, Zuluaga Gómez A. Hematomas renales secundarios a litotricia extracorporea por ondas de choque. Arch Esp Urol. 1997;50(7):767-771.

5. Akdas A, Turkeri LN, Ilker Y, Simsek F, Emerk K. Shortterm bioeffects of extracorporeal shockwave lithotripsy. J Endourol 1994;8(3):187-190.

6. Sen S, Erdem Y, Oymak O, Yalcin AU, Turgan C, Ersoy H, Bingol N, Tamer S. Effect of extracorporeal shock wave lithotripsy on glomerular and tubular functions. Int Urol Nephrol. 1996;28(3):309-313.

7. Willis LR, Evan AP, Connors BA, Reed G, Fineberg NS, Lingeman JA. Effects of extracorporeal shock wave lithotripsy to one kidney on bilateral glomerular filtration rate and PAH clearance in minipigs. J Urol. 1996;156(4):1502-1506.

8. Rutz-Danielczak A, Pupek-Musialik D, Raszeja-Wanic B. Effects of extracorporeal shock wave lithotripsy on renal function in patients with kidney stone disease. Nephron 1998;79(2): 162-166.

9. Liou LS, Streem SB. Long-term renal functional effects of shock wave lithotripsy, percutaneous nephrolithotomy and combination therapy: a comparative study of patients with solitary kidney. J Urol. 2001;166(1):36-37.

10. Sakkas G, Becopoulos T, Karayannis A, Drossos G, Giannopoulou K. Enzymatic evaluation of renal damage caused by different therapeutic procedures for kidney stone disease. Int Urol Nephrol 1995;27(6):669-77.

11. Karlsen S. Acute changes in kidney funtion following extracorporeal shock wave lithpotripsy for renal stones. $\mathrm{Br}$ J Urol 1991;67:241-245.

12. Pérez-Blanco FJ, Arrabal Martín M, Ocete Martín C, Arias Puerta JJ, García-Valdecasas Bernal J, Rodríguez Cuartero A, Zuluaga Gómez A. Glicosaminoglicanos urinarios tras litoricia extracorporea por ondas de choque en pacientes con litiasis renal. Arch Esp Urol. 2001;54(9): 875-883.

13. Stepanov VN, Perelman VM, Kadirov ZA, Istratov VG. Excretion urinarie de leucine aminopeptidase après extracorporel lithoticie avec choc vagues. J Urol 1995;101(4): 169-72.

Dr. M. Vírseda Chamorro

C/ Argentina, $4,1^{\circ}-1^{\mathrm{a}}$.

28027 Madrid

(Trabajo recibido el 18 noviembre de 2004) 